\title{
BMJ Open A stepped wedge, cluster controlled trial of an intervention to improve safety and quality on medical wards: the HEADS-UP study protocol
}

\author{
Samuel Pannick, ${ }^{1,2}$ lain Beveridge, ${ }^{2}$ Hutan Ashrafian, ${ }^{3}$ Susannah J Long, ${ }^{1,4}$ \\ Thanos Athanasiou, ${ }^{3}$ Nick Sevdalis ${ }^{5}$
}

To cite: Pannick S, Beveridge I, Ashrafian $\mathrm{H}$, et al. A stepped wedge, cluster controlled trial of an intervention to improve safety and quality on medical wards: the HEADS-UP study protocol. BMJ Open 2015;5: e007510. doi:10.1136/ bmjopen-2014-007510

- Prepublication history for this paper is available online. To view these files please visit the journal online (http://dx.doi.org/10.1136/ bmjopen-2014-007510).

Received 21 December 2014 Revised 1 April 2015 Accepted 10 April 2015

CrossMark

\begin{abstract}
${ }^{1}$ NIHR Imperial Patient Safety Translational Research Centre, Imperial College London, London, UK ${ }^{2}$ West Middlesex University Hospital NHS Trust, London, UK

${ }^{3}$ Department of Surgery \& Cancer, Imperial College London, London, UK ${ }^{4}$ St Mary's Hospital, Imperial College Healthcare NHS Trust, London, UK ${ }^{5}$ Centre for Implementation Science, Health Service \& Population Research Department, King's College
\end{abstract} London, London, UK

Correspondence to Dr Sam Pannick; S.Pannick@imperial.ac.uk

\begin{abstract}
Introduction: The majority of preventable deaths in healthcare are due to errors on general wards. Staff perceptions of safety correlate with patient survival, but effectively translating ward teams' concerns into tangibly improved care remains problematic. The Hospital Event Analysis Describing Significant Unanticipated Problems (HEADS-UP) trial evaluates a structured, multidisciplinary team briefing, capturing safety threats and adverse events, with rapid feedback to clinicians and service managers. This is the first study to rigorously assess a simpler intervention for general medical units, alongside an implementation model applicable to routine clinical practice.
\end{abstract}

Methods/analysis: 7 wards from 2 hospitals will progressively incorporate the intervention into daily practice over 14 months. Wards will adopt HEADS-UP in a pragmatic sequence, guided by local clinical enthusiasm. Initial implementation will be facilitated by a research lead, but rapidly delegated to clinical teams. The primary outcome is excess length of stay (a surplus stay of $24 \mathrm{~h}$ or more, compared to peer institutions' Healthcare Resource Groups-predicted length of stay). Secondary outcomes are 30-day readmission or excess length of stay; in-hospital death or death/readmission within 30 days; healthcareacquired infections; processes of escalation of care; use of traditional incident-reporting systems; and patient safety and teamwork climates. HEADS-UP will be analysed as a stepped wedge cluster controlled trial. With 7840 patients, using best and worst case predictions, the study would achieve between $75 \%$ and $100 \%$ power to detect a $2-14 \%$ absolute risk reduction in excess length of stay (two-sided $p<0.05$ ).

Regression analysis will use generalised linear mixed models or generalised estimating equations, and a time-to-event regression model. A qualitative analysis will evaluate facilitators and barriers to HEADS-UP implementation and impact.

Ethics and dissemination: Participating institutions' Research and Governance departments approved the study. Results will be published in peer-reviewed journals and at conference presentations.

Trial registration number: ISRCTN34806867.
Strengths and limitations of this study

- General wards typically generate the errors that lead to preventable deaths, but we know relatively little about how to improve the safety of care in this specific setting.

- This study will evaluate a new strategy to incorporate proactive team risk surveillance into routine care on general medical wards, with a facilitated organisational response: Hospital Event Analysis Describing Significant Unanticipated Problems (HEADS-UP).

- Mixed methods (quantitative and qualitative) will identify different aspects of the impact of HEADS-UP.

- With a relatively prolonged data collection period, the study is prey to unanticipated changes affecting participating sites.

\section{BACKGROUND}

Patient safety on medical wards

Despite an intense focus on healthcare safety in recent years, medical wards remain potentially perilous. Over $60 \%$ of medical wards' failings reach their patients, and $10 \%$ of those failings cause physical injury. ${ }^{1}$ These are not trivial problems: the general ward, more than any other care setting, generates the errors that lead to preventable deaths. ${ }^{2}$ Technical procedural failings are infrequent; more often, teams struggle to reliably monitor, assess and reassess their patients. ${ }^{2}$ Medical teams face increasing workloads, ${ }^{3}$ and concerns about the basic processes of ward care have been well publicised. ${ }^{4}$

Nonetheless, there is a relative paucity of specific literature on how to improve the quality and safety of care on medical wards. The same factors that contribute to serious errors in this setting-heterogeneous patient populations, geographic dispersion of medical teams, and frequent changes in staff, 
policies and procedures-have hampered the research necessary to address them. As a result, quality and safety interventions for medical patients are largely extrapolated from more structured clinical environments, that is, operating theatres and intensive care units (ICUs). Staff in medical units, however, would rapidly attest to the different challenges they face, compared to their colleagues in these other areas.

A recent review by our group established that interventions to improve medical ward care cluster across five themes: ${ }^{5}$

- Improving staffing levels and team composition;

- Improving communication and collaboration;

- Standardising care processes;

- Early recognition and treatment of the deteriorating patient;

- Improving patient safety climate.

Although the evidence base underpinning them is fragmented, these interventions are likely to have some positive impact on the quality and safety of care. Organisations increasingly adopt multifaceted improvement strategies that incorporate combinations of these five themes. ${ }^{6} 7$ These complex strategies are highly specific to their setting, and may be costly to implement. ${ }^{8}$ This limits their wider applicability, and a search for a more universally relevant tool is warranted.

\section{Frontline ward staff: a knowledgeable, but underused,} source of information

Frontline staff are a relatively untapped source of information about their own organisation and the effectiveness of its procedures and processes. It matters, what staff say: their perceptions correlate with patient survival. ${ }^{9}$ Moreover, finding the right tools to elicit staff concerns might facilitate change: organisations that seek out discomfiting insights, consciously listening to their staff, develop more holistic improvement strategies. ${ }^{10}$ Still, there are few descriptions of successful tools that systematically capture staff knowledge to improve clinical processes. Centralised incident reporting systems have major failings, ${ }^{11}{ }^{12}$ and physicians as a professional group do not engage with them. ${ }^{11}$ Improving reporting systems is a laudable goal, but additional methods are required to adequately detect and address problems before they lead to adverse events and patient harm. ${ }^{13}$

Importantly, the problem may lie with the available tools themselves, not their users. Ward staff are willing to disclose concerns about errors and potential errors to their peers, ${ }^{14}$ and incident reporting rates increase when clinicians are given more reporting options and receive feedback. ${ }^{15}$ Interactive schemes, visibly engaging entire teams to identify recurrent problems, may complement more traditional systems relying on individual reports. ${ }^{16} 17$ There is increasing interest in novel strategies to take advantage of team knowledge: for medical teams, the ward round may represent a specific opportunity to identify patient safety issues. ${ }^{18}$ Moreover, studies in paediatric centres suggest that patient-relevant process measures improve with a structured team approach to risk monitoring and situational awareness (eg, 'team huddles'). ${ }^{19}{ }^{20}$ It seems that a collective awareness of safety problems exists; exploiting it may have real benefits, beyond the typical reliance on individual efforts to intercept a catastrophic clinical deterioration, or report it after the event.

In our review, ${ }^{5}$ we identified no reports of simple interventions which (1) specifically targeted adult medical wards; (2) leading to team-wide engagement in a quality and safety initiative; with (3) rigorous assessment of patients' clinical outcomes. More complex interventions in this field have been tailored to the specific needs of individual clinical trial sites, or require resource-intensive support and mentoring, ${ }^{21}$ which limit their uptake in wider practice. Moreover, there are few reports of community hospitals replicating the experience of academic centres. ${ }^{22}$

The HEADS-UP (Hospital Event Analysis Describing Significant Unanticipated Problems) study that we describe here addresses these limitations with a tool that can be rapidly and easily adopted in any healthcare context. Our intervention aims to identify and mitigate systematic failings through daily whole-team recognition of clinical risks, facilitating improved team situational awareness, allied to a rapid organisational response.

\section{The HEADS-UP intervention}

A prompt-led team briefing (HEADS-UP) was designed to help multidisciplinary medical ward teams discuss clinical and administrative challenges, including adverse events, of the preceding $24 \mathrm{~h}$. The briefing can be led by any member of the team, regardless of seniority or role. HEADS-UP identifies ongoing concerns amenable to immediate intervention, as well as those requiring more detailed assessment and input from other departments. It also prompts team members to share information that they may not volunteer spontaneously. ${ }^{23}{ }^{24}$ Categories of prompts were guided by the published literature on the nature of common unintended events in medical departments ${ }^{1}$ (figure 1), with additional suggestions from ward clinicians regarding frequent or potentially serious lapses in care noticed in their clinical areas. At the request of participating physicians, a checklist-style pro-forma was used to facilitate speed of completion. We adopted a visual format similar to the World Health Organization's surgical safety checklist (figure 2). HEADS-UP summaries are regularly disseminated to participating teams, senior clinicians, managers and executives.

\section{Study aim and hypotheses}

The primary aim of this study is to assess the impact of the HEADS-UP briefing tool on clinically relevant patient outcomes. The secondary aim is to explore how changes in patient outcomes, if any, are mediated by changes in workplace climate and ward processes. 


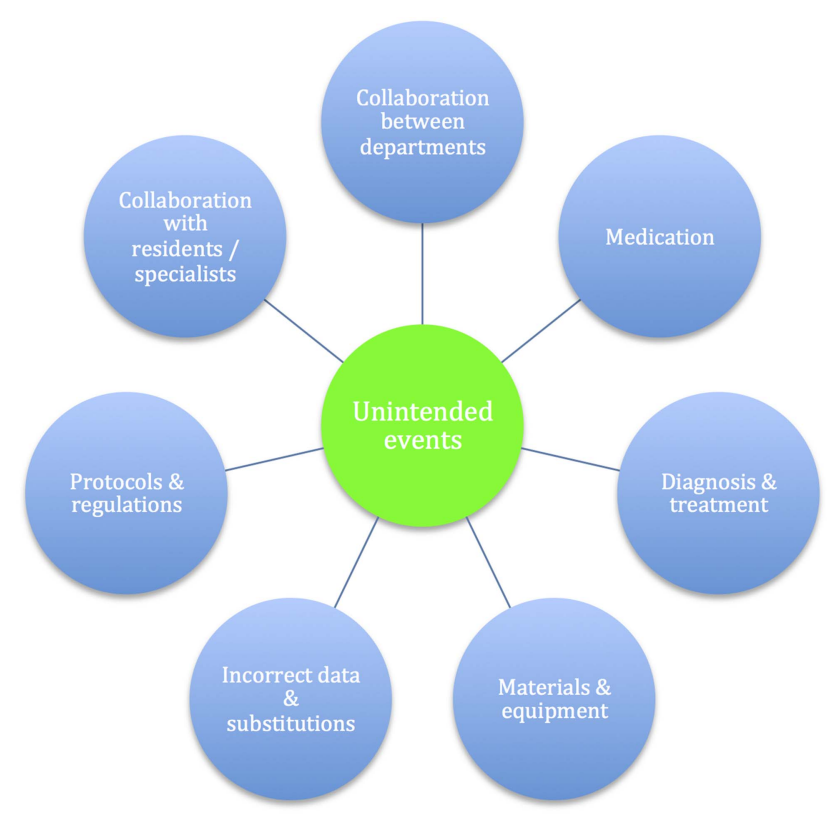

Figure 1 Categories of unintended events in internal medicine departments impacting negatively on patient care (after Lubberding et $a^{1}{ }^{1}$ ).

We hypothesised that:

- Team use of the HEADS-UP briefing would empower junior clinicians to voice concerns, improving their teams' situational awareness (an important factor in mitigating risks ${ }^{19}$ ) and their units' safety and teamwork climates;

- This would promote early team recognition of the deteriorating patient, and facilitate the process of escalation of care;

- Information generated by ward teams would both inform their own practice and prompt downstream service reorganisation;

- The combination of ward and support service improvement would improve clinical outcomes, with a dose-response relationship (ie, the better the tool is used in practice, the greater the benefit seen);

- An explicit focus on team-wide recognition of adverse events would improve engagement with existing incident-reporting systems, thus leading to an increase in formally reported incidents within wards implementing HEADS-UP.

\section{METHODS AND DESIGN \\ Study design and setting}

HEADS-UP is a prospective stepped wedge, cluster controlled trial, conducted in two hospitals in London, UK. The stepped wedge design involves the sequential introduction of the intervention to each of the clusters (in this case, wards) over time, with clusters progressively moving from the control group to the intervention group (figure 3). The staged implementation facilitated by the stepped wedge design is particularly helpful when

Figure 2 The HEADS-UP (Hospital Event Analysis Describing Significant Unanticipated Problems) team briefing tool. 
Figure 3 HEADS-UP (Hospital

Event Analysis Describing

Significant Unanticipated

Problems) stepped wedge cluster design.

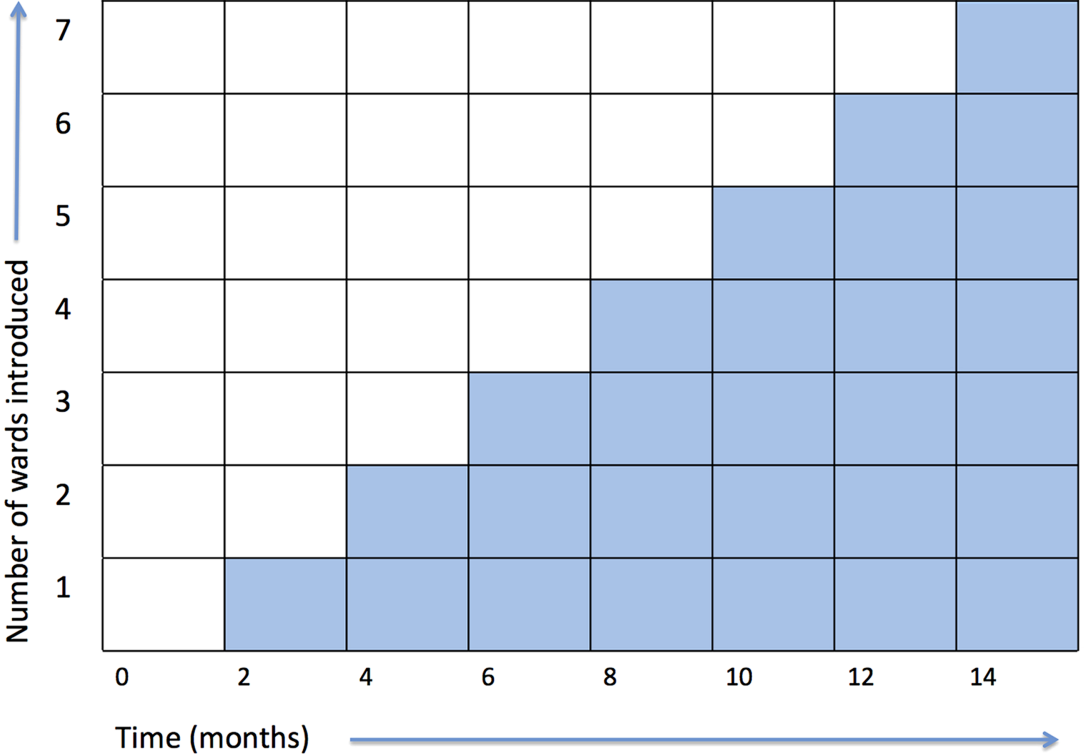

Time (months) simultaneous rollout of an intervention to all clusters is impractical, for example, for logistical or financial reasons. This trial design is used to evaluate interventions whose effects are predicted to be more beneficial than harmful, especially those interventions embedded in daily clinical practice. ${ }^{25}$ Stepped wedge designs are increasingly used in trials of interventions in acute care. ${ }^{26-29}$

The order in which clusters receive the intervention will be guided by logistical restrictions, and the de facto recognition of clinicians enthusiastic to introduce HEADS-UP to their wards. Although cluster randomisation (randomising the order in which the clusters receive the intervention) would be preferable, it is important to recognise the impact of willing early adopters who then lead their colleagues in implementing the intervention. ${ }^{30}$ They are likely to participate more extensively and follow through more rigorously and enthusiastically with the intervention than units at later stages of the intervention diffusion. ${ }^{31}$ Sufficient leadership and support from these early adopters will be needed to maximise the use of HEADS-UP in all the desired areas, much as the introduction of surgical checklists, for instance, has historically relied on strong clinical leadership. ${ }^{32}$ HEADS-UP is introduced to new clusters at two-monthly intervals.

The study is conducted at two sites. The first is a university-associated community general hospital. Clusters from this site are generated from the acute admissions and downstream medical (gastroenterology, respiratory and geriatric) wards. Each cluster comprises clinical areas that are physically linked, served by the same medical team, or both. This will help to limit contamination between groups. The second site is an academic hospital, where HEADS-UP will be implemented within the geriatric wards.

\section{Study population}

The study focuses on adult medical patients admitted to study wards between 2013 and 2015. To isolate the effect of the intervention, patient-level exclusion criteria will include:

- Time spent on the specified ward comprising less than $50 \%$ of the total inpatient stay;

- Discharge to a new skilled care facility or other hospital (ie, not the patient's address at the time of admission; discharge to a new facility typically incurs substantial delays, outside of the ward team's control);

- Multiple intrahospital ward transfers. A single transfer from the initial admissions unit to a downstream medical ward is permitted. One further transfer to an escalation area to facilitate discharge (whereby the patient spends less than $24 \mathrm{~h}$ in the escalation area immediately prior to their discharge home) is also permitted;

- Admission to the high-dependency unit, or ICU;

- Elective admission or direct admission from another hospital;

- Surgeon-directed care for more than $24 \mathrm{~h}$ during the inpatient stay.

\section{Outcome measures}

Primary outcome

- Excess length of stay (eLOS) - a surplus stay of $24 \mathrm{~h}$ or more, compared to peer institutions' Healthcare Resource Groups-predicted length of stay.

Secondary outcomes

- eLOS or 30-day readmission;

- In-hospital death or death/readmission within 30 days;

- Complications of care: hospital-acquired infections and pressure ulcers;

- Processes of escalation of care: use of the ICU outreach service, admissions to the ICU, and cardiac arrest calls;

- Staff engagement with the traditional web-based reporting system: number, and type (eg, patient falls 
or communication failures) of formally reported incidents;

- Patient safety climate: teamwork and safety subsections of the well validated Safety Attitudes Questionnaire (SAQ) ${ }^{33}$

We believed that tangible improvements in clinical outcomes and quality of care would be mediated by changes in ward processes, and ward safety and teamwork climates. Our chosen outcomes allow us to evaluate this hypothesis. The selected measures are similar to those used in other studies evaluating the quality of ward care, ${ }^{34}$ and have been assessed in a UK setting where appropriate. ${ }^{33}$ Table 1 describes specifically how the components of these outcomes correspond to the study hypotheses outlined earlier.
Intervention implementation

HEADS-UP implementation at ward level and clinical

engagement

Multidisciplinary ward teams will be asked to use HEADS-UP on a daily basis during the normal working week (Monday to Friday). Staffing and service provision are significantly reduced out of hours, and it was not deemed practical to incorporate a HEADS-UP briefing at night or at weekends within those constraints. One of the study leads at each site will supervise the initial use of the tool and answers any questions arising from it. The presence of a researcher will be documented when appropriate.

To maximise clinical engagement with this intervention, we aim to disrupt teams' existing working patterns

Table 1 Study outcomes and corresponding hypotheses evaluated within the HEADS-UP trial

\begin{tabular}{llll}
\hline & $\begin{array}{l}\text { Outcome } \\
\text { component }\end{array}$ & Relevant hypothesis & Rationale for outcome selection \\
\hline Primary outcome & Excess length of stay & $\begin{array}{l}\text { Improved clinical outcomes through } \\
\text { ward and support service } \\
\text { improvements }\end{array}$ & $\begin{array}{l}\text { Length of stay reflects efficient resource } \\
\text { use, and possibly quality of care. } \\
\text { of }{ }^{35} \text { Length } \\
\text { of stay varies substantially within } \\
\text { institutions, with wide differences between } \\
\text { the acute admissions unit and } \\
\text { downstream wards. Using excess length } \\
\end{array}$ \\
& & $\begin{array}{l}\text { of stay as an outcome increases study } \\
\text { power, facilitating statistical detection of a } \\
\text { meaningful change in outcome without } \\
\end{array}$ \\
& & $\begin{array}{l}\text { requiring an excessive number of wards } \\
\text { or data collection period. }\end{array}$
\end{tabular}

Secondary Mortality Improved clinical outcomes through Correlates with quality of care ${ }^{36}$ and may

outcomes:

clinical outcomes

Readmission

Complications of care

Improved situational awareness will mitigate patient risks

ward and support service

improvements

Improved clinical outcomes through

ward and support service

improvements
Secondary

outcomes:

processes of

care

Secondary

outcomes: staff

outcomes

Earlier team recognition of the

\section{Escalation of care}

.
deteriorating patient will facilitate processes underpinning escalation of care

Staff engagement
with traditional
reporting system
Safety and teamwork
climate

Team-wide recognition of adverse events will improve engagement with existing incident reporting systems

Empowerment of junior clinicians, with structured communication tool, will improve perceptions of safety and teamwork relate to performance in non-technical skill domains. $^{37}$

Need to confirm that improvements in hospital efficiency do not come at the expense of increased readmissions. $37 \%$ of medical readmissions are avoidable, ${ }^{38}$ a proportion that can be reduced with targeted quality improvement initiatives. $^{39-41}$

Agreement that these outcomes are appropriate patient safety indicators. ${ }^{42}$ Reliable reporting of these outcomes to confirm adherence to stringent centrally mandated targets. ${ }^{43} 44$

Multidisciplinary interventions, increasing team situational awareness, ${ }^{45}$ may address staff reluctance to appropriately escalate their concerns. ${ }^{24} 46$

More reports overall, with a lower contribution from reports of slips and falls, are associated with more positive safety culture and risk management ratings. ${ }^{47}$ Improved safety climate is associated with organisation-wide reduction in adverse events. ${ }^{48}$ SAQ scores previously assessed both in inpatient settings and in the UK. ${ }^{33}$

HEADS-UP, Hospital Event Analysis Describing Significant Unanticipated Problems; SAQ, Safety Attitudes Questionnaire. 
as little as possible; they will use HEADS-UP wherever it fits most naturally into their existing schedule. We anticipate that this will ordinarily be early in the day. The HEADS-UP tool itself acts as the written record of the briefing. Its format will remain stable, but further minor changes will be made at the request of individual ward teams to make it responsive to their specific needs. Successful improvement interventions reported elsewhere have adopted a similarly flexible approach. ${ }^{32} 49$

Support for the study will be sought from senior clinicians and executives with responsibility for clinical quality, safety and risk management at each site. Presentations to individual clinical teams will publicise the intervention prior to its introduction. No protected time will be available for HEADS-UP training, but the ideal format for each briefing will be discussed in departmental rounds, team meetings and with participating clinicians. The information shared during the daily HEADS-UP briefings may prompt a degree of reflective practice and immediate learning. In addition, a regular summary of the HEADS-UP events recorded from their clinical area will be given to each team. The format of this feedback again depends on the team's existing schedule; where possible, it will be incorporated into existing departmental educational or governance meetings, to place it in the appropriate context and minimise any additional time commitments. This feedback will emphasise the ongoing impact of the information gathered during the HEADS-UP briefings, highlighting any subsequent quality improvement work or changes to support services.

HEADS-UP summaries will also be shared with the governance committees responsible for the issues raised. Clinicians and managers already accountable for quality and safety in these clinical areas will be expected to use the information appropriately to guide resource allocation, and make changes to routine processes or procedures as they see fit. No specific guidance will be issued as to how the HEADS-UP data will be used. We aim to evaluate how, in practice, the intervention will generate changes in clinical services. However, specific safety concerns and significant adverse events raised through HEADS-UP briefings will be emphasised to the responsible clinical team or governance body in order that they take appropriate action.

\section{Fidelity of implementation and confounding factors}

The quality of HEADS-UP implementation will be evaluated primarily through the daily briefing documentation, recording the team members present, number and type of concerns raised, and the decisions taken as a result. A narrative diary, describing the qualitative impact of HEADS-UP, as well as the downstream quality improvement work and service changes arising from it, will complement the quantitative outcomes. The narrative account will also describe the observation of a number of HEADS-UP briefings, and the extent to which they held true to the perceived ideal in terms of participants, timeliness, focus and intent. The individual, team and organisational barriers and facilitators to the effective implementation of HEADS-UP will be assessed. Together with the objective outcomes listed in table 1, and the staff survey (SAQ) data, this will complete a mixed methods analysis of the HEADS-UP programme.

Staff workload and patient casemix are likely to be the two predominant confounding factors for this nonrandomised study. Ward bed occupancy rates will be documented from routine hospital administration systems, and staff perceptions of workload assessed periodically with the validated NASA-Task Load Index (NASA-TLX) tool. ${ }^{50}$ Patient casemix will be tracked with an updated version of the Charlson comorbidity index..$^{51} 52$

\section{Blinding}

Professionals implementing the intervention are not blinded to the ward's assignment group: this is not possible, given the nature of the intervention. Extraction of the clinical outcome measures will be performed primarily by administrative staff not involved in the study (as part of their ordinary duties), who will be blinded to intervention groups.

\section{Data management}

Data will be extracted directly from hospital administrative systems, with a monthly assessment by administrative staff to confirm its reliability. Anonymised data, where appropriate, will be held securely on password-protected hospital intranet systems. Given the nature of the intervention, the time scale of the study, and the extraction of outcomes from existing administrative systems, no data monitoring committee is required. The summarised trial data set will be held by SP, and disseminated to local clinical and managerial healthcare providers as required after the study, with no contractual limitations.

\section{Sample size, power calculation and analysis}

In the 33 months preceding the study, local data from six of the seven anticipated clusters described a median of 522 patients matching our inclusion/exclusion criteria each month (range 316-722). Mean eLOS rates ranged from $5.6 \%$ to $51.5 \%$.

Cluster controlled trials also require an estimation of the intraclass correlation coefficient (ICC); this is complex, and observed ICCs rarely match their predicted values. ICG estimation should take into account the results of previous studies, as well as the anticipated numbers of individuals and clusters in the index study. Greater numbers of individuals in a study reduce the width of the ICC confidence interval, mitigating the effect of a relatively small number of clusters. ${ }^{53}$ In addition, clinical outcome measures tend to have lower ICCs than process measures. ${ }^{53}$ The observed ICC for falls in a multifactorial intervention on elderly care wards was only $0.007 .{ }^{54}$ However, ICCs for length of stay and appropriateness of stay in trials of inpatient care pathways were an order of magnitude higher. ${ }^{55}$ We therefore adopted the more conservative ICC 
estimate of 0.06 . In any case, the power under a stepped wedge cluster randomised trial is relatively insensitive to ICC underestimation, compared to a parallel cluster design. ${ }^{56}$ Similarly, stepped wedge trial power is relatively insensitive to variations in the coefficient of variation. ${ }^{57} \mathrm{In}$ addition, in our best-case prediction the coefficient of variation approached the level at which adjusting for variable cluster size has a negligible impact on sample size, even in parallel cluster trials. ${ }^{58}$ No adjustment for coefficient of variation was therefore required.

Given the variation in baseline outcomes in our local data set, we then estimated the study's power to detect a 1 standard deviation reduction in eLOS on the wards with the highest and lowest baseline outcome rates. With 7840 patients in the trial (560 patients/month), and two-sided $\mathrm{p}<0.05$, the study would achieve $100 \%$ power to detect a $14 \%$ absolute risk reduction. At worst, in the ward with the lowest baseline outcome rate, it would achieve $75 \%$ power to detect a $2.3 \%$ absolute risk reduction. We therefore propose that the study's power to detect a $2-14 \%$ absolute risk reduction lies between $75 \%$ and $100 \%$. With this complex trial design, statistical uncertainty in power calculations is not unusual. The recent protocol of a large stepped wedge cluster randomised trial similarly produced a range within which its power might lie. ${ }^{29}$ Experts also advocate more widespread recognition of the inherent limitations of statistical power thresholds. ${ }^{59}$

The stepped wedge design compares outcomes in each cluster before and after the introduction of the intervention. Overall differences in outcomes between preintervention and postintervention periods will be reported. Primary analysis will be on an intention-totreat basis, with a separate prespecified per-protocol analysis of those units implementing the intervention with high fidelity. Analyses will use the patient-level data described above, clustered within 'units', using random effects to model the correlation between individuals within the same cluster. Generalised linear mixed models, or generalised estimating equations, will form the basis of the analysis. ${ }^{57}$ Results will also be assessed using a time-to-event regression model. Underlying temporal trends (including seasonal trends) will be accounted for. We will evaluate any interaction effect between intervention group and 'duration of HEADS-UP implementation' to see if the intervention exerts an incremental effect over time. There will be a prespecified analysis excluding patients coded for palliative care, to further isolate the effect of the intervention. Sensitivity analyses will judge whether primary outcome results remain unchanged when other patient groups (eg, those spending less than $50 \%$ of their stay on the specified ward) are included. No interim analyses are planned, nor is a formal health economics analysis.

\section{RESULTS: PILOT IMPLEMENTATION}

During a 6-week pilot period, participating clinicians honed the format of the HEADS-UP tool, ensuring it could be completed quickly and accurately. Subsequent iterations of the HEADS-UP tool incorporated further feedback from clinical staff and clinical governance teams on the content and use of the HEADS-UP briefing. This largely resulted in an increased focus on the outcomes of the briefing, namely the actions to be taken and which team member would be responsible for them.

The introduction of the tool was supervised initially by a physician researcher $(\mathrm{SP})$. When led by a clinician, the HEADS-UP briefing typically took between 5 and 8 min to complete. Clinical teams' use of the tool was deliberately unsupervised for the latter half of the pilot period, to gauge whether it was suitably concise and relevant to be used in practice without the presence of the researcher. The HEADS-UP briefing was completed unsupervised on $80 \%$ of working days. Taken together, these data suggest that the HEADS-UP trial is feasible.

\section{TRIAL STATUS}

Data collection is ongoing.

\section{DISCUSSION}

This stepped wedge cluster controlled trial will assess the impact of a simple intervention to improve quality and safety on medical wards. The design of the HEADS-UP tool, as well as its proposed implementation, intentionally minimises the disparity between the trial setting and daily clinical practice. This will make the study results immediately applicable to broad swathes of healthcare settings. We hope to obtain conclusive evidence for the success or failure of the intervention, but with a relatively prolonged data collection period, the study is prey to unanticipated systemic changes outside of our control. There is also a tension between effective HEADS-UP promotion, essential for adequate staff engagement, and contamination of the control groups. In addition, downstream interventions arising from the HEADS-UP process may impact multiple wards, regardless of their participation in HEADS-UP. The narrative record will help highlight where this may have been the case, and contextualise the impact of the tool.

In summary, HEADS-UP offers a novel, rapid wholeteam analysis of clinical and administrative challenges, including adverse events, at ward level. This prospective trial will identify whether HEADS-UP is a useful addition to existing safety systems, and broader lessons for the implementation of safety and quality interventions within the complex medical ward environment.

Twitter Follow Sam Pannick at @Sam_Pannick

Acknowledgements We would like to thank Catriona Barron for her work on data collection for this study.

Contributors SP conceived the intervention, designed the study and the implementation model, and drafted the manuscript. IB and SJL designed the implementation model. HA and TA coordinated the statistical analysis. NS designed the study and the implementation model. All authors contributed to, read, and approved the final manuscript. 
Funding This study was funded by the National Institute for Health Research, via the NIHR Imperial Patient Safety Translational Research Centre (http:// www.cpssq.org), grant number RDPSC79560. Additional funding was provided by the West Middlesex University Hospital NHS Trust. Neither institution had any other involvement with the design of the study; the authors were independent. SP and IB are supported by the West Middlesex University Hospital NHS Trust; SP, HA and TA by Imperial College London; SL by Imperial College Healthcare NHS Trust; and NS' research is supported by the National Institute for Health Research (NIHR) Collaboration for Leadership in Applied Health Research and Care South London at King's College Hospital NHS Foundation Trust. The views expressed are those of the authors and not necessarily those of the NHS, the NIHR or the Department of Health.

Competing interests NS is the director of London Training \& Safety Solutions Ltd, which delivers team interventions, assessment and training to hospitals in the UK and internationally on a consultancy basis.

Ethics approval Permission for the study was sought from the relevant Research \& Development authority at each participating institution. Both authorities approved the study as a service development initiative not requiring formal ethical evaluation. The authors were initially advised by the Office for Regulatory Compliance at the Imperial College Academic Health Science Centre that registration with a clinical trial database would not be required, given the nature of the intervention. This decision was reviewed again, and the study registered - prior to completion of data collection - in the ISRCTN registry (http://www.isrctn.com), identifier ISRCTN34806867.

Provenance and peer review Not commissioned; externally peer reviewed.

Open Access This is an Open Access article distributed in accordance with the terms of the Creative Commons Attribution (CC BY 4.0) license, which permits others to distribute, remix, adapt and build upon this work, for commercial use, provided the original work is properly cited. See: http:// creativecommons.org/licenses/by/4.0/

\section{REFERENCES}

1. Lubberding S, Zwaan L, Timmermans DR, et al. The nature and causes of unintended events reported at 10 internal medicine departments. J Patient Saf 2011;7:224-31.

2. Hogan H, Healey F, Neale G, et al. Preventable deaths due to problems in care in English acute hospitals: a retrospective case record review study. BMJ Qual Saf 2012;21:737-45.

3. The Royal College of Physicians. The medical registrar: empowering the unsung heroes of patient care. London: RCP, 2013. http://www. rcplondon.ac.uk/sites/default/files/future-medical-registrar_1.pdf

4. Francis R. Report of the Mid Staffordshire NHS Foundation Trust Public Inquiry. London: Stationery Office, 2013.

5. Pannick S, Beveridge I, Wachter RM, et al. Improving the quality and safety of care on the medical ward: a review and synthesis of the evidence base. Eur J Intern Med 2014;25:874-87.

6. Beckett DJ, Inglis M, Oswald S, et al. Reducing cardiac arrests in the acute admissions unit: a quality improvement journey. BMJ Qual Saf 2013;22:1025-31.

7. McAlister FA, Bakal JA, Majumdar SR, et al. Safely and effectively reducing inpatient length of stay: a controlled study of the Genera Internal Medicine Care Transformation Initiative. BMJ Qual Saf 2014;23:446-56.

8. Auerbach AD, Sehgal NL, Blegen MA, et al. Effects of a multicentre teamwork and communication programme on patient outcomes: results from the Triad for Optimal Patient Safety (TOPS) project. BMJ Qual Saf 2012;21:118-26.

9. Tvedt C, Sjetne IS, Helgeland J, et al. An observational study: associations between nurse-reported hospital characteristics and estimated 30-day survival probabilities. BMJ Qual Saf 2014;23:757-64.

10. Dixon-Woods M, Baker R, Charles $\mathrm{K}$, et al. Culture and behaviour in the English National Health Service: overview of lessons from a large multimethod study. BMJ Qual Saf 2014;23:106-15.

11. Farley DO, Haviland A, Champagne S, et al. Adverse-eventreporting practices by US hospitals: results of a national survey. Qual Saf Health Care 2008;17:416-23.

12. Shojania KG. The frustrating case of incident-reporting systems. Qual Saf Health Care 2008;17:400-2.

13. Olsen S, Neale G, Schwab K, et al. Hospital staff should use more than one method to detect adverse events and potential adverse events: incident reporting, pharmacist surveillance and local realtime record review may all have a place. Qual Saf Health Care 2007;16:40-4.

14. Weingart SN, Ship AN, Aronson MD. Confidential clinician-reported surveillance of adverse events among medical inpatients. J Gen Intern Med 2000;15:470-7.

15. Evans SM, Smith BJ, Esterman A, et al. Evaluation of an intervention aimed at improving voluntary incident reporting in hospitals. Qual Saf Health Care 2007;16:169-75.

16. Sujan MA, Ingram C, McConkey $\mathrm{T}$, et al. Hassle in the dispensary: pilot study of a proactive risk monitoring tool for organisational learning based on narratives and staff perceptions. BMJ Qual Saf 2011;20:549-56.

17. Lear R, Vincent C, Van Herzeele I, et al. Structured team self-report of intraoperative error can identify obstacles to safe surgery. Jt Comm J Qual Patient Saf 2013;39:480.

18. Lamba AR, Linn K, Fletcher KE. Identifying patient safety problems during team rounds: an ethnographic study. BMJ Qual Saf 2014;23:667-9.

19. Brady PW, Muething S, Kotagal U, et al. Improving situation awareness to reduce unrecognized clinical deterioration and serious safety events. Pediatrics 2013;131:e298-308.

20. Goldenhar LM, Brady PW, Sutcliffe KM, et al. Huddling for high reliability and situation awareness. BMJ Qual Sa 2013;22:899-906.

21. Hansen LO, Greenwald JL, Budnitz T, et al. Project BOOST: effectiveness of a multihospital effort to reduce rehospitalization. J Hosp Med 2013;8:421-7.

22. Zaubler TS, Murphy K, Rizzuto L, et al. Quality improvement and cost savings with multicomponent delirium interventions: replication of the hospital elder life program in a community hospital. Psychosomatics 2013;54:219-26.

23. Blackman J, Walsgrove J, Gunawardena I. A study examining rates of medical staff recognition of pressure ulceration in hospital inpatients. Postgrad Med J 2013;89:258-61.

24. Kennedy TJ, Regehr G, Baker GR, et al. Preserving professional credibility: grounded theory study of medical trainees' requests for clinical support. BMJ 2009;338:b128.

25. Mdege ND, Man MS, Taylor Nee Brown CA, et al. Systematic review of stepped wedge cluster randomized trials shows that design is particularly used to evaluate interventions during routine implementation. J Clin Epidemiol 2011;64:936-48.

26. Kitson AL, Schultz TJ, Long $\mathrm{L}$, et al. The prevention and reduction of weight loss in an acute tertiary care setting: protocol for a pragmatic stepped wedge randomised cluster trial (the PRoWL project). BMC Health Serv Res 2013;13:299.

27. Poldervaart JM, Reitsma JB, Koffijberg $\mathrm{H}$, et al. The impact of the HEART risk score in the early assessment of patients with acute chest pain: design of a stepped wedge, cluster randomised trial. BMC Cardiovasc Disord 2013;13:77.

28. Fuller C, Michie S, Savage J, et al. The Feedback Intervention Trial (FIT) -improving hand-hygiene compliance in UK healthcare workers: a stepped wedge cluster randomised controlled trial. PLoS ONE 2012;7:e41617.

29. Pearse R. Enhanced peri-operative care for high-risk patients $(\mathrm{EPOCH})$ trial: a stepped wedge cluster randomised trial of a quality improvement intervention for patients undergoing emergency laparotomy. http://www.epochtrial.org/docs/EPOCH\%20Protocol\% 20revision\%20V2\%200\%20280414.pdf (accessed 10 Dec 2014).

30. Wilson CB. Adoption of new surgical technology. BMJ 2006;332:112-14

31. Frankel A, Grillo SP, Pittman M, et al. Revealing and resolving patient safety defects: the impact of leadership WalkRounds on frontline caregiver assessments of patient safety. Health Serv Res 2008;43:2050-66.

32. World Alliance for Patient Safety. WHO surgical safety checklist and implementation manual. http://www.who.int/patientsafety/ safesurgery/ss_checklist/en/ (accessed 15 May 2014).

33. Sexton JB, Helmreich RL, Neilands TB, et al. The Safety Attitudes Questionnaire: psychometric properties, benchmarking data, and emerging research. BMC Health Serv Res 2006;6:44.

34. Timmermans MJ, van Vught AJ, Wensing $M$, et al. The effectiveness of substitution of hospital ward care from medical doctors to physician assistants: a study protocol. BMC Health Serv Res 2014:14:43.

35. Svendsen ML, Ehlers LH, Andersen G, et al. Quality of care and length of hospital stay among patients with stroke. Med Care 2009;47:575-82.

36. Jha AK, Orav EJ, Li Z, et al. The inverse relationship between mortality rates and performance in the Hospital Quality Alliance measures. Health Aff (Millwood) 2007;26:1104-10. 
37. Curry LA, Spatz E, Cherlin E, et al. What distinguishes topperforming hospitals in acute myocardial infarction mortality rates? A qualitative study. Ann Intern Med 2011;154:384-90.

38. Donze J, Aujesky D, Williams D, et al. Potentially avoidable 30-day hospital readmissions in medical patients: derivation and validation of a prediction model. JAMA Intern Med 2013;173:632-8.

39. Torisson G, Minthon L, Stavenow L, et al. Multidisciplinary intervention reducing readmissions in medical inpatients: a prospective, nonrandomized study. Clin Interv Aging 2013;8:1295-304.

40. Wong FKY, Ho MM, Yeung S, et al. Effects of a health-social partnership transitional program on hospital readmission: a randomized controlled trial. Soc Sci Med 2011;73:960-9.

41. Amarasingham R, Patel PC, Toto $\mathrm{K}$, et al. Allocating scarce resources in real-time to reduce heart failure readmissions: a prospective, controlled study. BMJ Qual Saf 2013;22: 998-1005.

42. Savitz LA, Jones CB, Bernard S. Quality indicators sensitive to nurse staffing in acute care settings. In: Henriksen K, Battles JB, Marks ES, et al., eds. Advances in patient safety: from research to implementation (volume 4: programs, tools, and products). Agency for Healthcare Research and Quality: Rockville, MD, 2005.

43. NHS England. Zero tolerance-guidance on the post infection review. http://www.england.nhs.uk/ourwork/patientsafety/zerotolerance/ (accessed 31 Mar 2014).

44. NHS England. 2014/15 Clostridium difficile infection objectives http://www.england.nhs.uk/ourwork/patientsafety/associatedinfections/clostridium-difficile/ (accessed 31 Mar 2014).

45. Pronovost $\mathrm{P}$, Berenholtz $\mathrm{S}$, Dorman $\mathrm{T}$, et al. Improving communication in the ICU using daily goals. J Crit Care 2003;18:71-5.

46. McNeill G, Bryden D. Do either early warning systems or emergency response teams improve hospital patient survival? A systematic review. Resuscitation 2013;84:1652-67.

47. Hutchinson A, Young TA, Cooper KL, et al. Trends in healthcare incident reporting and relationship to safety and quality data in acute hospitals: results from the National Reporting and Learning System. Qual Saf Health Care 2009;18:5-10.
48. Mardon RE, Khanna K, Sorra J, et al. Exploring relationships between hospital patient safety culture and adverse events. J Patient Saf 2010;6:226-32.

49. Bosk CL, Dixon-Woods M, Goeschel CA, et al. Reality check for checklists. Lancet 2009;374:444-5.

50. Hart SG. NASA-Task Load Index (NASA-TLX); 20 Years Later. Proceedings of the Human Factors and Ergonomics Society 50th Annual Meeting, 904-908; Santa Monica: HFES, 2006. [as cited at http://humansystems.arc.nasa.gov/groups/t|x/t|xpublications.html]

51. Charlson ME, Pompei P, Ales KL, et al. A new method of classifying prognostic comorbidity in longitudinal studies: development and validation. J Chronic Dis 1987:40:373-83.

52. Health and Social Care Information Centre. Indicator Specification: Summary Hospital-level Mortality Indicator methodology (version 1.17). http://www.hscic.gov.uk/media/11151/Indicator-SpecificationSummary-Hospital-level-Mortality-Indicator-methodology/pdf/SHMI Specification.pdf (accessed 9 Oct 2014).

53. Eldridge S. Sample size calculations for cluster randomised trials. http://www.newton.ac.uk/seminar/20110815152516051 (accessed 11 Dec 2014).

54. Cumming RG, Sherrington C, Lord SR, et al. Cluster randomised trial of a targeted multifactorial intervention to prevent falls among older people in hospital. BMJ 2008;336:758-60.

55. Kul S, Vanhaecht K, Panella M. Intraclass correlation coefficients for cluster randomized trials in care pathways and usual care: hospital treatment for heart failure. BMC Health Serv Res 2014;14:84.

56. Hemming K, Lilford R, Girling AJ. Stepped-wedge cluster randomised controlled trials: a generic framework including parallel and multiple-level designs. Stat Med 2015;34:181-96.

57. Hussey MA, Hughes JP. Design and analysis of stepped wedge cluster randomized trials. Contemp Clin Trials 2007;28:182-91.

58. Eldridge SM, Ashby D, Kerry S. Sample size for cluster randomized trials: effect of coefficient of variation of cluster size and analysis method. Int J Epidemiol 2006;35:1292-300.

59. Campbell MJ. Doing clinical trials large enough to achieve adequate reductions in uncertainties about treatment effects. $J R$ Soc Med 2013;106:68-71. 